Pacific Journal of Mathematics

ON SUBGROUPS OF AN ABELIAN GROUP MAXIMAL 


\title{
ON SUBGROUPS OF AN ABELIAN GROUP MAXIMAL DISJOINT FROM A GIVEN SUBGROUP
}

\author{
J. D. REID
}

1. Introduction. In [3], J. M. Irwin has introduced the concept of a high subgroup of an abelian group ( $A$ is high in $G$ if $A$ is maximal in $G$ with respect to the property $\left.A \cap\left(\cap_{n} n G\right)=0\right)$. Irwin and, subsequently, Irwin and Walker [4] have also considered $N$-high subgroups $A$ of $G$ ( $A$ is maximal in $G$ with respect to $A \cap N=0$ ). Among the properties of high subgroups is their purity in $G$ ([1], [3] for p-groups, [3] for torsion groups and [4] for arbitrary abelian groups). In [5], S. Khabbaz has given a short proof of a theorem which implies the purity of high subgroups of a $p$-group. Irwin [3] raises the question of characterizing subgroups $H$ of a group $G$ for which every $H$-high subgroup is pure in $G$.

In this paper we consider pairs $(H, M)$ of subgroups of an abelian group $G$ with $M$ maximal disjoint from $H$ in $G$ and ask what happens if $M$ is not pure in $G$. The resulting information allows us to answer Irwin's question in various special cases. In particular we obtain the purity of high subgroups of arbitrary abelian groups and a generalization of the theorem of Khabbaz referred to above. We then consider various related questions and obtain a generalization of a theorem of Žuravskir [7] on the splitting of mixed abelian groups.

Throughout the paper, $G$ will denote an abelian group, $H$ a subgroup of $G$ and $M$ a subgroup of $G$ maximal with respect to $M \cap H=0$. Following Irwin [3] we say that $M$ is $H$-high in $G$. For any subgroup $K$ of $G$ and prime $p, K_{p}$ denotes the set of all elements of $K$ whose orders are a power of $p$, and $K[p]$ is the set of elements of $K_{p}$ whose orders are $\leqq p$. The torsion subgroup of a group $K$ will occasionally be denoted by $K_{t}$. For $x \in G$ we denote by $h_{p}(x)=$ $\max \left[n \mid x \in p^{n} G\right]$ the height of the element $x$ at $p$ in $G$. Curly brackets denote the subgroup generated by the sets and elements inside. In particular, if $M$ is a subgroup of $G$ and $x \in G$ then $\{M, x\}$ is the subgroup of $G$ generated by $M$ and $x$. The set of rational integers will be denoted by $Z$, direct sums by $\oplus$ and not necessarily direct sums by + .

2. The main theorem. We remark first that if $M$ is $H$-high in $G$ then $M$ is neat in $G$ (cf. [2, pp. 91-92]); i.e, $M \cap p G=p M$ for each prime $p$. It is also easy to see that $G[p]=M[p] \oplus H[p]$ for any $p$.

Received March 4, 1962, and in revised for December, 12, 1962. This paper was written while the author was an ONR research associate. Support for this research by the ONR is gratefully acknowledged. 
THEOREM 2.1. Let $M$ be an H-high subgroup of $G$. Then either $M$ is pure in $G$ or there exists a prime $p$ and elements $\boldsymbol{m} \in M$, $h \in H[p]$ such that

$$
h_{p}(\mathbf{m})=h_{p}(h)<h_{p}(h-\boldsymbol{m}) .
$$

Proof. Suppose that $M$ is not pure in $G$. Then there exist. equations $n w=v$ with $n \in Z, w \in G, v \in M$ which have no solution $w \in M$. Among all such equations, let $n x=y(x \in G, y \in M)$ be one for which $n$ is least positive. It is not hard to see that minimality of $n$ implies that $n$ is a power of some prime $p$, say $n=p^{r}$. By neatness of $M, r>1$ and $p^{r} x=p m_{1}, m_{1} \in M$ so that $p\left(p^{r-1} x-m_{1}\right)=0$. Thus $p^{r-1} x-$ $m_{1} \in G[p]$ and since $G[p]=M[p] \oplus H[p]$, we have

$$
p^{r-1} x-m_{1}=m+h \quad(m \in M[p], h \in H[p]) .
$$

Suppose now that $h_{p}(h) \geqq r-1$. Then $h=p^{r-1} z$ for some $z \in G$ which, from (1) and minimality of $r$ yields $m_{1}+m=p^{r-1}(x-z)=p^{r-1} m_{2}$ for some $m_{2} \in M$. But this gives $p^{r-1} x=p^{r-1} m_{2}+h$ or, $p^{r} x=p^{r} m_{2}=y$ contrary to the choice of $r$. Thus $h_{p}(h)<r-1$ and $p^{r-1} x-\left(m+m_{1}\right)=$ $h$. With $\boldsymbol{m}=-\left(m+m_{1}\right)$ we now have

$$
h_{p}(\boldsymbol{m})=h_{p}(h)<h_{p}(h-\boldsymbol{m})
$$

and the theorem follows.

CoRollary 2.2. If, for each $p$, either $M \cong p G$ or $H_{p}$ is divisible, then $M$ is pure in $G$.

Proof. Neatness of $M$ and $M \cong p G$ give $M=p M$. Thus, for each prime $p, m \in M$ and $h \in H_{p}$, either $h_{p}(m)=\infty$ or $h_{p}(h)=\infty$.

The author is indebted to the referee for the proof of Theorem 2.1 given above a proof which is shorter and less complicated than the author's original. The original proof, however, had a corollary which, at the suggestion of the referee, we include here. The proof requires that we outline the proof of Theorem 2.1 given originally. Therefore we state the result as

Proposition 2.3. Let $H$ be a subgroup of $G$ such that $H_{t}=G_{t}$ and let $M$ be $H$-high in $G$. If $M$ is not pure in $G$ then there exists a prime $p$ and elements $m \in M, h \in H[p]$ such that

$$
0=h_{p}(m)=h_{p}(h)<h_{p}(h-m) .
$$

Proof (in outline). Let $p^{r}, x$ and $y$ be as in the proof of Theorem 
2.1. Then $\{M, x\} \cap H \neq 0$ so there exist nonzero elements of $H$ of the form $u+n x$ with $u \in M$ and $n$ a positive integer. Let $c$ be the least positive integer such that $m+c x \in H, m+c x \neq 0$ for some $m \in M$. Then one can show that $c=p^{k}$ for some $k<r$, and with $h=m+p^{k} x$ we have $h \in H|p|$ and

$$
h_{p}(m)=h_{p}(h)<h_{p}(h-m)=k
$$

At this point we have a proof of Theorem 2.1, since we have not yet used the hypothesis $H_{t}=G_{t}$. Now, $p^{r-1}(p x)=y$ so that by choice of $y$ there exists $v \in M$ such that $p^{r-1} v=y=p^{r} x$. Hence $p^{r-1}(p x-v)=$ 0 and $r-1>0$. Using $G_{t}=H_{t}$ we conclude that $p x-v \in H$. It is clear that $p x-v \neq 0$ so that $k=1$. Thus $(*)$ gives $h_{p}(m)=h_{p}(h)=0$ as required.

\section{Centers of purity.}

Definition 3.1. A subgroup $H$ of an abelian group $G$ will be called a Center of Purity in $G$ if every $H$-high subgroup of $G$ is pure in $G$.

Several classes of centers of purity can be obtained from the following proposition which is a corollary to Theorem 2.1.

Proposition 3.2. If there exists a homomorphism $f$ defined on $G$ such that

(i) $H_{t} \subset$ kernel $f$

(ii) $h_{p}(m)=h_{p}(f(m))$ for all $m \in M$ and primes $p$ then $M$ is pure in $G$.

Proof. For any prime $p, m \in M$ and $h \in H_{p}$ we have

$$
h_{p}(m)=h_{p}(f(m))=h_{p}(f(m-h)) \geqq h_{p}(m-h)=h_{p}(h-m)
$$

so that the condition in Theorem 1 alternative to purity of $M$ cannot hold. Hence $M$ is pure.

The following corollary generalizes the theorem of Khabbaz [4] referred to in the introduction.

COROLlary 3.3. Let $G$ be a p-group and put $p^{\infty} G=\bigcap_{n} p^{n} G$, $p^{\infty+1} G=0$. Then any subgroup $H$ of $G$ such that $p^{s} G \supseteqq H \supseteqq p^{s+1} G$ for some $s, 0 \leqq s \leqq \infty$, is a center of purity in $G$.

Proof. Let $f$ be the canonical homomorphism $f: G \rightarrow G / H$. Then $p^{s+1}(G / H)=0$ (by definition if $s=\infty$ ) so $h_{p}(f(x)) \leqq s$ for all $x \in G$, $x \notin H$. Suppose $p^{u} f(y)=f(x)$ for some $u \in Z$ and $x \notin H$. Then $p^{u} y+$ $h=x$ for some $h \in H$. Since $H \leqq p^{s} G, u \leqq s$ and $u<\infty$ there exists 
$w \in G$ such that $p^{u} w=h$. Hence, $p^{u}(y+w)=x$. Thus $h_{p}(x) \geqq h_{p}(f(x))$. The other inequality being obvious we have $h_{p}(x)=h_{p}(f(x))$ for all $x \in G, x \notin H$ and the corollary follows.

CoRollary 3.4. For any abelian group $G$ and subgroup $H$ of $G$, if $H_{t} \leqq \bigcap_{n} n G$, then $H$ is a center of purity in $G$. In particular, high subgroups are pure and torsion free subgroups are centers of purity. If the maximal torsion subgroup of $G$ is divisible, every subgroup of $G$ is a center of purity in $G$.

Proof. As in Corollary 3.3 with $f$ the canonical homomorphism $f: G \rightarrow G / H_{t}$.

One can ask with Irwin [3] for necessary and sufficient conditions on a subgroup $H$ of a group $G$ in order that $H$ be a center of purity in $G$. We have not been able to find such conditions. In particular, we know of no centers of purity in a $p$-group other than those listed in Corollary 3.3 above but have not been able to show that there are no others. In one case, however, a decisive answer is readily obtained. We denote by $T$, in what follows, the maximal torsion subgroup of $G$.

LEMma 3.5. If $T \cong H$ then $H$ is a center of purity in $G$ if and only if for all $g \in G$ and primes $p$, the conditions $\{g\} \cap H=0$ and $h_{p}(g)=0$ imply $h_{p}(g+t)=0$ for all $t \in T$.

Proof. If the condition is satisfied, then $H$ is a center of purity by Proposition 2.3. Conversely, if $H$ is a center of purity in $G$ and $g \in G$ such that $\{g\} \cap H=0$ and $h_{p}(g)=0$ for some $p$ then there exists a subgroup $M$ of $G$ maximal disjoint from $H$ and containing $g$. For $t \in T$, if $h_{p}(t)>0$ it is clear that $h_{p}(g+t)=h_{p}(g)=0$. Suppose then that $t \in T$ and $h_{p}(t)=0$. We can write $t=t_{p}+t^{\prime}$ where $t_{p}$ has order $p^{l}$ for some $l \geqq 0$ and the order of $t^{\prime}$ is prime to $p$. Then $h_{p}\left(t^{\prime}\right)=\infty$ so that $h_{p}(t)=h_{p}\left(t_{p}\right)=0$. Clearly also $h_{p}(g+t)=h_{p}\left(g+t_{p}\right) \geqq k$ say. Let $x \in G$ such that $p^{k} x=g+t_{p}$. Then, with $e=k+l$, we have $p^{e} x=p^{l} g$. By purity of $M$ there exists $m \in M$ such that $p^{e} m=p^{l} g$. Hence $p^{l}\left(p^{k} m-g\right)=0$. Now since $T \subseteq H$ and $M \cap H=0$ we have $p^{k} m=g$ so that $k=0$ by hypothesis on $g$. Thus, $h_{p}\left(g+t_{p}\right)=h_{p}(g+t)=$ 0 as required.

Definition 3.6. A subgroup $H$ of $G$ containing $T$ will be called a special center of purity if $H$ is a center of purity and there exists $x \in G$ such that $x \neq 0,\{x\} \cap H=0$. A mixed group $G$ is said to be properly mixed if $0 \neq T \neq G$.

THEOREM 3.7. For a properly mixed group $G$ the following are 
equivalent:

(i) $T$ is divisible.

(ii) Every subgroup of $G$ is a center of purity in $G$.

(iii) $G$ contains a special center of purity.

Proof. (i) implies (ii) by Corollary 3.4. (ii) implies (iii) since if (ii) holds and $G$ is properly mixed, then $T$ is a special center of purity. To show that (iii) implies (i), let $H$ be a special center of purity in $G$. If $T$ is not divisible then $T_{p}$ is not divisible for some $p$ so that there exists $t \in T_{p}$ such that $h_{p}(t)=0$. Let $x \in G,\{x\} \cap H=0$ and $x \neq 0$ and put $g=p x+t$. Clearly $\{g\} \cap H=0$ and $h_{p}(g)=0$. However. $h_{p}(g-t) \geqq 1$. This contradicts Lemma 3.5 and completes the proof.

4. Reduction theorems. If $M$ is maximal disjoint from $H$ in $G$, we consider here circumstances under which we can reduce the problem of the purity of $M$ in $G$ to an analogous problem in a subgroup of $G$ or in a factor group of $G$. Again, the location of $T$ with respect to $H$ plays a role.

THeOREM 4.1. Let $M$ and $R$ be 'subgroups of $G$ such that $G=$ $M+R$. Then

(i) $M$ is maximal disjoint in $G$ from a subgroup $H \subseteq R$ if and only if $M \cap R$ is maximal disjoint from $H$ in $R$.

(ii) If $M \cap R$ is pure in $R$ then $M$ is pure in G. Conversely if $T \subseteq R$ and $M$ is pure in $G$ then $M \cap R$ is pure in $R$.

Proof. (i) If $M$ is maximal disjoint in $G$ from $H \subseteq R$ and $r \in R$, $r \notin M \cap R$ then $\{M, r\} \cap H \neq 0$. Hence there exist $m \in M, a \in Z$ such that $m+a r \in H, m+a r \neq 0$. Since $H \subseteq R$, we have $m+a r \in R$ so that $m \in M \cap R$. Thus $\{M \cap R, r\} \cap H \neq 0$.

Conversely, if $M \cap R$ is maximal disjoint from $H$ in $R$ and $g \in G$, $g \notin M$ we have $g=m+r$ for some $m \in M, r \in R$ by hypothesis. Now, $g \notin M$ implies that $r \notin M \cap R$ so that $\{M \cap R, r\} \cap H \neq 0$. Let $m_{1} \in M$, $b \in Z$ such that $h=m_{1}+b r \in H, h \neq 0$. Then $m_{1}+b g=h+b m$ so $m_{1}-b m+b g=h \in H, h \neq 0, m_{1}-b m \in M$; i.e. $\{M, g\} \cap H \neq 0$. Now since $0=M \cap R \cap H=M \cap H, M$ is maximal disjoint from $H$ as required.

(ii) If $M \cap R$ is pure in $R$ and $n g=m \in M$, let $g=m_{1}+r, m_{1} \in M$, $r \in R$. Then $m=n g=n m_{1}+n r$ so $m-n m_{1}=n r \in M \cap R$. By purity of $M \cap R$ in $R$ there exists $m_{2} \in M \cap R$ such that $n m_{2}=m-n m_{1}$. Hence $n\left(m_{1}+m_{2}\right)=m$ with $m_{1}+m_{2} \in M$ as required.

Conversely, if $T \subseteq R$ and $M$ is pure in $G$, suppose $n r=m \in M \cap R$ for some $n \in Z, r \in R$. By purity of $M$ in $G$ there exists $m_{1} \in M$ such that $n m_{1}=m$. Then $n\left(m_{1}-r\right)=0$ so that $m_{1}-r \in T \leqq R$; i.e. $m_{1} \in M \cap R$. Thus $M \cap R$ is pure in $R$. 
THEOREM 4.2. Suppose $T \subseteq H$ and let $M$ be maximal disjoint from $H$ in $G$. Then the following are equivalent:

(i) $M$ is pure in $G$

(ii) $(M+T) / T$ is pure in $G / T$

(iii) $(M+T) / T$ is maximal disjoint from $H / T$ in $G / T$.

Proof. It is well known (cf. [2, p. 94]) that if $M$ is pure in $G$ then $\{M, T\}$ is pure in $G$ and in our case the converse is true since $\{M, T\}=M \oplus T$. Now, since $T$ is pure in $G, M+T$ is pure in $G$ if and only if $M+T / T$ is pure in $G / T$ so that (i) and (ii) are equivalent. Also, since $G / T$ is torsion free, if $M+T / T$ is maximal disjoint from $H / T$ then $M+T / T$ is pure in $G / T$ so that (iii) implies (ii). Finally assume that $M$ is pure in $G$ and let $g+T \in G / T, g+T \notin M+T / T$. Then $g \notin M$ so there exist $m \in M, a \in Z$ such that $m+a g \in H, m+a g \neq$ 0 . If $m+a g-t \in T$ say $b t=0$. Then $b m=-b a g$ and by purity of $M$ in $G$ there exists $m_{1} \in M$ such that $b a m_{1}=b m$. Then $b\left(a m_{1}-m\right)=0$ so $a m_{1}=m$ since $M \cap T=0$. Now we have $t=m+a g=a\left(m_{1}+g\right) \in T$ whence $m_{1}+g \in T$. But this contradicts $g+T \notin M+T / T$. We conclude that $(m+a g)+T \in H / T, m+a g+T \neq T$ so that, disjointness of $M+T / T$ from $H / T$ being clear, $M+T / T$ is maximal disjoint from $H / T$ in $G / T$.

5. On the splitting of mixed groups. As an immediate consequence of Theorem 4.2 and Proposition 3.2 we have

Proposition 5.1. Let $T$ be the maximal torsion subgroup of the mixed group $G$. Then the following are equivalent:

(i) $G=M \oplus T$.

(ii) $M$ is maximal disjoint from $T$ in $G$ and pure in $G$.

(iii) $M$ is maximal disjoint from $T$ in $G$ and the natural mapping $\nu: G \rightarrow G / T$ is height preserving on $M$; i.e. $h_{p}(m)=h_{p}(\nu(m))$ for all $m \in M$, and all primes $p$.

As a result, there exist groups at the opposite end of the spectrum from centers of purity; i.e. since there exist nonsplitting mixed groups, we have

COROLLARY 5.2. There exist groups $G$ containing subgroups $H$ such that, if $M$ is maximal disjoint from $H$ in $G$ then $M$ is not pure.

One is tempted to try to use Proposition 5.1 to obtain splitting criteria for mixed groups in terms of the structure of the groups. If, for example, $G$ contains a subgroup $M$ maximal disjoint from $T$ and $p$-divisible for all $p$ for which $T_{p} \neq 0$ then $M$ is pure by Theorem 2.1 and hence $G$ splits. A necessary condition for such a situation is 
of course that $G / T$ be $p$-divisible for all $p$ for which $T_{p} \neq 0$. Just this condition has recently been considered by V. S. Žuravskiì ([6], [7]).

Although we fail to apply Proposition 5.1 we can point out a generalization of the result of Zuravskil and, since the proof is quite short, it may be worthwhile to include this here. First, we observe

LEMMA 5.3. Let $G$ be a mixed group and $R$ the subgroup of $G$ generated by a complete system of representatives of $G \bmod T$. If $R$ splits, $R=S \oplus R \cap T$, then $G$ splits, $G=S \oplus T$.

The proof is immediate. Now, for a mixed group $G$, we say that $G$ satisfies the maximal element condition if each coset of $T$ in $G$ contains an element $x$ such that $h_{p}(x)=h_{p}(x+T)$ for all $p$. Evidently (either directly or by Proposition 5.1) this is a necessary condition for the splitting of $G$. Let $\pi$ be the set of primes $p$ for which $T_{p} \neq 0$.

THEOREM 5.4. Let $G$ be a mixed group and $T$ its maximal torsion subgroup. Suppose that

(i) $G$ satisfies the maximal element condition.

(ii) $G / T$ is $p$-divisible for all $p \in \pi$.

(iii) $\bigcap_{p \in \pi} \bigcap_{n} p^{n} T$ is bounded.

Then $G$ splits.

Proof. In each coset of $T$ in $G$ select an element $x$ such that $h_{p}(x)=h_{p}(x+T)$ for all $p$. Then, by (ii) $h_{p}(x)=\infty$ for all $p \in \pi$. Let $R$ be the subgroup of $G$ generated by the elements so selected. Then it is clear that $R \cap T \subseteq \bigcap_{p \in \pi} \bigcap_{n} p^{n} T$ and hence, by (iii) $R \cap T$ is bounded. Thus $R$ splits, so $G$ splits also.

The case treated by Žuravskii is that in which $T$ is $p$-primary and $G / T$ is rank one. He also constructs an example [7, p. 380, Theorem 3.4] of a nonsplitting mixed group $G$ satisfying (i) and (ii) (with $T$ a primary group and $G / T$ of rank one) but not (iii) so in this sense, condition (iii) is necessary. We remark that, as stated, [7, p, 380, Theorem 3.4] seems to say that given conditions (i) and (ii), the condition (iii) is necessary and sufficient for the splitting of $G$, but this is obviously false and, equally obviously (from the proof of the theorem) not what the author intended.

\section{BIBLIOGRAPHY}

1. B. Charles, Étude sur les sous-groupes d'un groupe abélien, Bull. Soc. Math. France, 88 (1960), 217-227.

2. L. Fuchs, Abelian groups, Budapest, Hungarian Academy of Sciences, 1958.

3. J. M. Irwin, High subgroups of abelian torsion groups, Pacific J. Math., 11 (1961), 1375-1384. 
4. J. M. Irwin and E. A. Walker, On N-high subgroups of abelian groups, Pacific J. Math., 11 (1961), 1363-1374.

5. S. Khabbaz, On a theorem of Charles and Erdelyi, Bull. Math. Soc. France, 89 (1961), 103-104.

6. V. S· Zuravskiř, On the splitting of certain mixed abelian groups, Mat. Sb. (N.S.) 48 (90) (1959), 499/508.

7. - Generalization of some criteria for splitting of mixed abelian groups, Mat. Sb. (N.S.) 51 (93) (1960), 377-382.

YAle UNiversity AND AmHerst College 


\title{
PACIFIC JOURNAL OF MATHEMATICS
}

\author{
EDITORS
}

\author{
Ralph S. Phillips \\ Stanford University \\ Stanford, California \\ M. G. Arsove \\ University of Washington \\ Seattle 5 , Washington
}

\author{
J. DugunduI \\ University of Southern California \\ Los Angeles 7, California
}

Lowell J. Paige

University of California

Los Angeles 24, California

\section{ASSOCIATE EDITORS}

E. F. BECKENBACH

T. M. CHERRY
D. DERRY
M. OHTSUKA

H. L. ROYDEN

E. SPANIER
E. G. STRAUS

F. WOLF

\section{SUPPORTING INSTITUTIONS}

UNIVERSITY OF BRITISH COLUMBIA CALIFORNIA INSTITUTE OF TECHNOLOGY UNIVERSITY OF CALIFORNIA MONTANA STATE UNIVERSITY UNIVERSITY OF NEVADA NEW MEXICO STATE UNIVERSITY OREGON STATE UNIVERSITY UNIVERSITY OF OREGON OSAKA UNIVERSITY UNIVERSITY OF SOUTHERN CALIFORNIA
STANFORD UNIVERSITY

UNIVERSITY OF TOKYO

UNIVERSITY OF UTAH

WASHINGTON STATE UNIVERSITY

UNIVERSITY OF WASHINGTON

AMERICAN MATHEMATICAL SOCIETY CALIFORNIA RESEARCH CORPORATION SPACE TECHNOLOGY LABORATORIES NAVAL ORDNANCE TEST STATION

Mathematical papers intended for publication in the Pacific Journal of Mathematics should be typewritten (double spaced), and the author should keep a complete copy. Manuscripts may be sent to any one of the four editors. All other communications to the editors should be addressed to the managing editor, L. J. Paige at the University of California, Los Angeles 24, California.

50 reprints per author of each article are furnished free of charge; additional copies may be obtained at cost in multiples of 50 .

The Pacific Journal of Mathematics is published quarterly, in March, June, September, and December. Effective with Volume 13 the price per volume (4 numbers) is $\$ 18.00$; single issues, $\$ 5.00$. Special price for current issues to individual faculty members of supporting institutions and to individual members of the American Mathematical Society: $\$ 8.00$ per volume; single issues \$2.50. Back numbers are available.

Subscriptions, orders for back numbers, and changes of address should be sent to Pacific Journal of Mathematics, 103 Highland Boulevard, Berkeley 8, California.

Printed at Kokusai Bunken Insatsusha (International Academic Printing Co., Ltd.), No. 6, 2-chome, Fujimi-cho, Chiyoda-ku, Tokyo, Japan.

PUBLISHED BY PACIFIC JOURNAL OF MATHEMATICS, A NON-PROFIT CORPORATION

The Supporting Institutions listed above contribute to the cost of publication of this Journal, but they are not owners or publishers and have no responsibility for its content or policies. 


\section{Pacific Journal of Mathematics}

\section{Vol. 13, No. 2 \\ April, 1963}

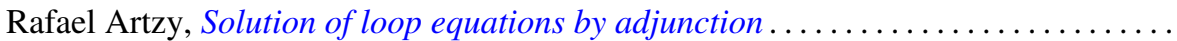

Earl Robert Berkson, A characterization of scalar type operators on reflexive

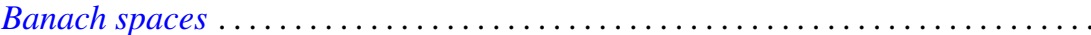

Mario Borelli, Divisorial varieties

365

Raj Chandra Bose, Strongly regular graphs, partial geometries and partially

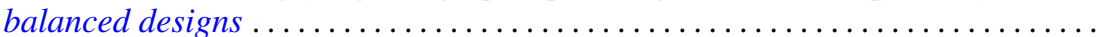

389

R. H. Bruck, Finite nets. II. Uniqueness and imbedding ............... 421

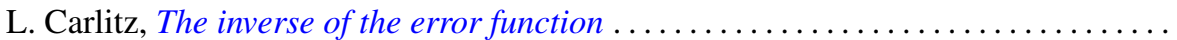

Robert Wayne Carroll, Some degenerate Cauchy problems with operator

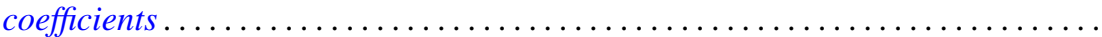

Michael P. Drazin and Emilie Virginia Haynsworth, A theorem on matrices of 0 's

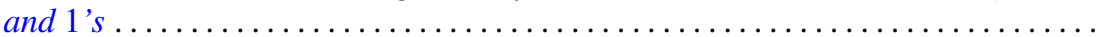

Lawrence Carl Eggan and Eugene A. Maier, On complex approximation .......... James Michael Gardner Fell, Weak containment and Kronecker products of group

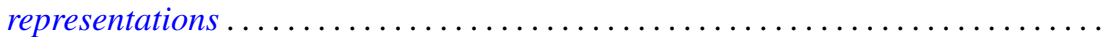

Paul Chase Fife, Schauder estimates under incomplete Hölder continuity

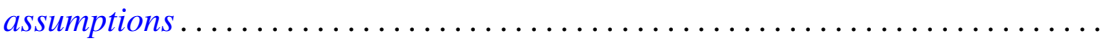

Shaul Foguel, Powers of a contraction in Hilbert space ...................

Neal Eugene Foland, The structure of the orbits and their limit sets in continuous

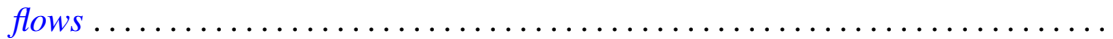

Frank John Forelli, Jr., Analytic measures . . . . . . . . . . . . . . . . . . . . . 563

Robert William Gilmer, Jr., On a classical theorem of Noether in ideal theory ....... P. R. Halmos and Jack E. McLaughlin, Partial isometries .

Albert Emerson Hurd, Maximum modulus algebras and local approximation in

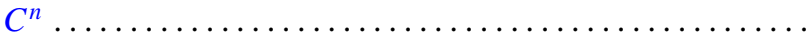

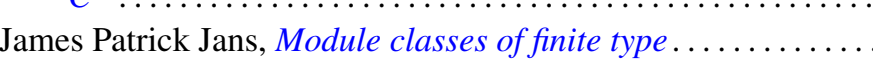

Betty Kvarda, On densities of sets of lattice points ...

H. Larcher, A geometric characterization for a class of discontinuous groups of linear fractional transformations .

John W. Moon and Leo Moser, Simple paths on polyhedra .

T. S. Motzkin and Ernst Gabor Straus, Representation of a point of a set as sum of

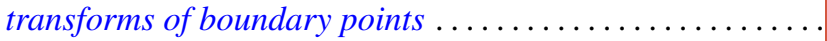

Rajakularaman Ponnuswami Pakshirajan, An analogue of Kolmogorov's three-series theorem for abstract random variables ...............

Robert Ralph Phelps, Čebyšev subspaces of finite codimension in $C(X)$...

James Dolan Reid, On subgroups of an Abelian group maximal disjoint from a given subgroup ...

William T. Reid, Riccati matrix differential equations and non-oscillation criteria for associated linear differential systems .................

Georg Johann Rieger, Some theorems on prime ideals in algebraic number fields ...

Gene Fuerst Rose and Joseph Silbert Ullian, Approximations of functions on the

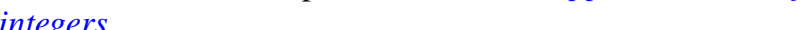

F. J. Sansone, Combinatorial functions and regressive isols . . . . . . . . . . 703

Leo Sario, On locally meromorphic functions with single-valued moduli . . . . . . . 709

Takayuki Tamura, Semigroups and their subsemigroup lattices.

Pui-kei Wong, Existence and asymptotic behavior of proper solutions of a class of second-order nonlinear differential equations . . ........... 